\title{
Evidence for a neuromuscular circuit involving hypothalamic Interleukin- 6 signaling in the control of skeletal muscle metabolism
}

\section{Carlos Katashima}

State University of Campinas

Thayana Micheletti

University of Campinas

Alexandre Moura-Assis

University of Campinas

\section{Rodrigo Gaspar}

State University of Campinas

\section{Ludger Goeminne}

Ecole Polytechnique Fédérale de Lausanne (EPFL) https://orcid.org/0000-0002-8285-7766

\section{Renata Braga}

University of Campinas

\section{Barbara Crisol}

\section{University of Campinas}

\section{Vagner Ramon Silva}

https://orcid.org/0000-0003-4793-1075

\section{Camila Ramos}

University of Campinas

\section{Alisson Rocha}

University of São Paulo

\section{Mariana Tavares}

University of Campinas

\section{Fernando Simabuco}

University of Campinas

\section{Patricia Pazos}

University of Santiago de Compostela

\section{David Gonzalez-Touceda}

University of Santiago de Compostela

\section{Sulay Tovar}

University of Santiago de Compostela https://orcid.org/0000-0001-7813-6520

\section{Maria García}


University of Santiago de Compostela

José Neto

University of São Paulo

\section{Rui Curi}

University of São Paulo

\section{Sandro Hirabara}

Yale School of Medicine

\section{Patrícia Brum}

University of São Paulo

\section{Patrícia Prada}

University of Campinas

\section{Leandro de Moura}

State University of Campinas

Jose Pauli

University of Campinas

\section{Adelino Silva}

University of São Paulo (USP)

\section{Dennys Cintra}

State University of Campinas

\section{Licio Velloso}

State University of Campinas https://orcid.org/0000-0002-4806-7218

Eduardo Ropelle ( $\nabla$ eduardoropelle@gmail.com )

University of Campinas

\section{Letter}

Keywords: skeletal muscle, hypothalamic Interleukin-6, metabolism

Posted Date: July 29th, 2021

DOl: https://doi.org/10.21203/rs.3.rs-737420/v1

License: (a) (i) This work is licensed under a Creative Commons Attribution 4.0 International License. Read Full License 


\section{Abstract}

Hypothalamic interleukin-6 (IL6) exerts a broad metabolic control, including energy expenditure ${ }^{1}$, food consumption ${ }^{2}$, glucose homeostasis ${ }^{2}$, etc. Here we demonstrated that Interleukin-6 (IL6) activates the ERK1/2 pathway in the ventromedial hypothalamus (VMH), stimulating AMPK/ACC signaling and fatty acid oxidation in mice skeletal muscle. Bioinformatics analysis revealed that the hypothalamic IL6/ERK12 axis is closely associated with firing-rate-related genes in the hypothalamus and with fatty acid oxidation- and mitochondrial-related genes in skeletal muscle of genetically diverse BXD mice strains and humans. Using surgical denervation, pharmacological approaches, and transgenic mice, we showed that the hypothalamic IL6/ERK1/2 pathway requires the a2-adrenergic pathway to modify the fatty acid skeletal muscle metabolism. To address the physiological relevance of these findings, we demonstrated that this neuromuscular circuitry is required to underpin AMPK/ACC signaling activation and fatty acid oxidation post-exercise. Once the selective downregulation of IL6 receptor in VMH abolished the effects of exercise to sustain AMPK and ACC phosphorylation and fatty acid oxidation in the muscle postexercise. Altogether, these data demonstrated that IL6/ERK axis in VMH controls fatty acid metabolism in mice skeletal muscle.

\section{Main Text}

Neuronal activity in the ventromedial hypothalamus $(\mathrm{VMH})$ controls energy expenditure ${ }^{3}$ and skeletal muscle metabolism, including fatty acid oxidation and glucose uptake ${ }^{4,5}$. Hormones and neuropeptides, such as leptin and orexin, can trigger signals into VHM nucleus to control the muscle metabolism in murine models $\mathrm{s}^{4,6}$. Thus, we sought to determine the existence of a neuromuscular circuit involving hypothalamic IL6 action in VMH nucleus toward the skeletal muscle metabolism, driving the lipid metabolism.

Interaction graph by using Genotype-Tissue Expression (GTEx) human database ${ }^{7}$ from 81 individuals revealed a positive association (red lines) between hypothalamic IL 6 gene expression and lipid metabolism- and mitochondrial-related genes in the skeletal muscle (Figure 1A). No correlation was observed between the hypothalamic IL 6 gene and glucose metabolism- and protein synthesis-related genes (Figure 1A). Hypothalamic IL6 gene expression showed a positive correlation (red lines) with lipid metabolism-related genes (Figure 1B - upper panels) and negative correlation (green lines) with lipid biosynthesis-related genes (Figure 1B - lower panels). These data were confirmed by a two-factor analysis (Figure 1C).

We next employed analyses using a system data from the BXD mouse genetic reference population ${ }^{8}$, geared towards multiscalar integration of traits. This data set also showed positive interaction between hypothalamic $/ 16$ gene expression and gene-sets involved in fatty acid oxidation in skeletal muscle (red lines). Conversely, negative interaction (green lines) was observed among hypothalamic $/ 16$ gene expression and expression of some markers of lipid biosynthesis in skeletal muscle of BXD mice (Figures $1 \mathrm{D}$ and $\mathrm{E})$. No interaction was found among hypothalamic $/ / 6$ gene expression and glucose metabolism 
or protein synthesis-related genes (Figure 1D). The principal component analysis (PCA) confirmed a strong positive correlation between hypothalamic $/ / 6$ levels and lipid oxidation- and mitochondrial-related genes in muscle (Figure 1F). The heat map graph revealed that specific strains of BXD mice with low levels of hypothalamic 116 gene expression (BXD63, 90 and 65a) displayed high levels of transcripts related to skeletal muscle lipid biosynthesis (Figure 1G). Conversely, BXD families with high levels of hypothalamic $/ 16$ gene expression (BXD43, 99, and 84) present high lipid oxidation- and mitochondrialrelated genes in skeletal muscle (Figure 1G). The two-factor loadings plot confirmed that the hypothalamic $/ / 6$ gene expression is positively correlated with fatty acid oxidation markers (Acadsb, Cpt1c, Mrp/41, Pcca, and Uqcrc) and showed a negative correlation with markers of lipid biosynthesis (Srebp1c and Scd1) in the skeletal muscle of strains of genetically diverse BXD mice (Figure $1 \mathrm{H})$.

Beyond the genetic variation and correlative analyses, we also tested our hypothesis experimentally by using recombinant IL6 icv microinjection in C57BL6/j mice (Figure 1I). Recombinant IL6 (200ng) increased the oxygen consumption (Figure $1 \mathrm{~J}$ ) and stimulated critical markers involved in fatty acid oxidation in the soleus muscle, including ACC ${ }^{\text {Ser79/Ser212 }}$ and AMPK ${ }^{\text {Thr172 }}$ phosphorylation (Figure 1K) and $C p t 1 \beta$ and Ppara gene expression (Figure 1L). Recombinant IL6 icv administration induced transient fatty acid oxidation in the soleus muscle, peaking 3 hours after the central injection (Figure $1 \mathrm{M}$ ). The mass spectrometry analysis was performed using the same experimental design to deeply assess the hypothalamic IL6 impact on the skeletal muscle lipid metabolism. The central action of IL6 markedly reduced the amount of fraction of saturated (C15:0, C16:0, C17:0, and C18:0), monosaturated (C18:1, C18:1 $t$ and $C 20: 1)$, and polyunsaturated (18:2, 18:3 and 20:3) fractions of fatty acids in the skeletal muscle (Figure 1N-P and S1A). Collectively, these initial data revealed that the hypothalamic IL6 action modulates the lipid metabolism in the skeletal muscle.

Several pieces of evidence are connecting the $\mathrm{VMH}$ nucleus and muscle metabolism ${ }^{5,9}$. In addition, it has been demonstrated that neurons expressing steroidogenic factor- 1 (SF-1) ${ }^{10}$ or extracellular signalregulated kinase 1/2 (ERK1/2) ${ }^{11}$ in VMH elicit signals from the central nervous system toward the skeletal muscle. Curiously, IL6 stimulates both SF-1 expression and ERK1/2 phosphorylation in human ${ }^{12}$ and murine ${ }^{13}$ cell lines. To explore the possibility of IL6 to modulates targets such as SF-1 and/or ERK1/2 expressing neurons in VMH, we first evaluated the presence of IL6 receptor (IL6R) in neurons of hypothalamic nuclei of mice. Double-staining analysis revealed that IL6R (red) is spread in neurons (green) of different hypothalamic nuclei of mice (Figure 2A, S1B, and S1C), including in the VMH (Figure 2B). We also found IL6R in non-neuronal cells (yellow arrows) in VMH (Figure 2B). Nevertheless, the presence of IL6R in SF-1 neurons of VMH was not clearly observed (Figure S1D). We also found the presence of ERK1/2 phosphorylation (green) in IL6R-expressing cells (red) in VMH of mice (Figure S2A). Based on these findings and previous studies that reported that hypothalamic ERK signaling plays a critical role in controlling sympathetic flow ${ }^{14,15}$, we hypothesized that the hypothalamic IL6/ERK axis in VMH could control the skeletal muscle lipid metabolism. 
After that, we evaluated the capacity of exogenous IL 6 to activate ERK1/2 in the hypothalamus. First, we observed that hypothalamic IL6 gene expression was strongly associated with firing-rate-related transcripts in mice and humans (Figure S2B and C). Indeed, the recombinant IL6 icv microinjection stimulated ERK1/2 phosphorylation in the whole hypothalamus (Figure $2 \mathrm{C}$ ) and specifically in VMH of mice (Figure 2D). We observed that IL6 also stimulated ERK1/2 phosphorylation in Neuro 2A cells (Figure $2 \mathrm{E})$. These data revealed the potential of IL 6 in stimulating neuronal activity and ERK $1 / 2$ phosphorylation, as previously reported ${ }^{16,17}$.

To test if hypothalamic IL6/ERK1/2 axis is involved in the skeletal muscle lipid metabolism, PD98059, a MEK/ERK signaling pharmacological inhibitor or saline, were icv administrated 30 minutes before the icv microinjection of IL6 recombinant, as detailed in the experimental design (Figure 2F). Notably, PD98059 abolished hypothalamic IL6-induced ACC and AMPK phosphorylation (Figure 2G) and fatty acid oxidation (Figure $2 \mathrm{H}$ ) in soleus muscle. We also evaluated the interaction between hypothalamic ERK1/2 signaling and lipid metabolism in skeletal muscle of humans. The interactome analysis and heat map graph confirmed a consistent association between hypothalamic ERK1 and ERK2 gene expression and several genes involved in fatty acid metabolism in human skeletal muscle (Figures 2I-K), but not glucose metabolism or protein synthesis (Figure 2l). Altogether, bioinformatics and experimental data demonstrated that the hypothalamic IL6/ERK axis controls AMPK/ACC signaling and fatty acid oxidation in the soleus muscle. In the same line, leptin modulates muscle metabolism through ERK $1 / 2$ activation in VMH of rats ${ }^{11}$.

Next, we investigated the involvement of the sympathetic nervous system in this neuromuscular circuitry. The left hindlimb of mice was denervated, and the contralateral paw was used as control. Two days after the surgeries (cannula implant and denervation), the animals received recombinant IL6 (200ng) icv microinjection, and the soleus muscles were removed, as demonstrated in the experimental design (Figure 3A). Interestingly, the surgical denervation reduced the ability of the central action of IL6 to phosphorylates ACC and AMPK in the soleus muscle of mice (Figure 3B), demonstrating that the hypothalamic IL 6 requires the nervous projections to alter the muscle metabolism.

Notably, a-adrenergic signals mediate the ACC phosphorylation and fatty acid oxidation in the skeletal muscle in response to intrahypothalamic injections of leptin ${ }^{6}$ and the synthetic inhibitor of fatty acid synthase, $\mathrm{C} 75^{18}$. Based on this information, we sought to determine the involvement of a-adrenergic signaling in this neuromuscular circuitry. Interactome analysis revealed that in humans, the hypothalamic $/ / 6$ gene is positively correlated with expression of gene-set linked to alpha-adrenergic signaling in the skeletal muscle, including ADRA2C, CREM, ADCY3, PDE4B, PDE4D, and AK4 genes (Figure $3 C$ ). These data were also confirmed by Pearson's correlations (Figure 3D) and two-component analyses (Figure 3E). In the BXD cohort, we found that a2 adrenoceptors subunits (Adra2c, 2a and 2b), but not a1 subunits, were highly expressed in skeletal muscle of strains with high expression of hypothalamic //6 (Figure 3SA-C). These transcriptomic analyses led us to investigate the involvement of a2 adrenoceptors subunits as critical components to connect the central IL6 action with the skeletal muscle metabolism. For this, mice lacking both a2A and a2C adrenoceptors (a2AC KO) were generated. Wild-type and a2AC 
$\mathrm{KO}$ mice received an icv microinjection of IL6 recombinant and the soleus muscle were removed for analyses 3 hours later (Figure 3F). Interestingly, the central action of IL6 increased ACC and AMPK phosphorylation in the muscle of wild-type, but not, in double-knockout mice (Figure 3G). Furthermore, icv IL6 administration failed to stimulate fatty acid oxidation in the skeletal muscle of a2AC KO mice (Figure $3 \mathrm{H})$. No difference was observed in the IL6 serum levels between wild-type and a2AC KO mice, or after icV IL6 microinjection (Figure 3I). Together, these data demonstrate that a2A and a2C receptors subunits are involved in the terminal sympathetic flow in muscle fibers, integrating the hypothalamic IL6 effects.

Thereafter, we sought to evaluate this neuromuscular circuitry under some physiological conditions. It has been demonstrated that a single bout of exercise can underpin subsequent resting lipid oxidation for many hours in humans ${ }^{19-21}$. However, the mechanism by which our organism maintains the fatty acid oxidation in muscle fiber post-exercise remains largely unknown. Intriguingly, high levels of IL6 were observed in the hypothalamus of fish ${ }^{22}$, mice ${ }^{23}$, and in the central nervous system of humans ${ }^{24}$ postexercise. Thus, we hypothesized that hypothalamic IL6 could be involved in the fatty acid oxidation in the skeletal muscle post-exercise session. To address this issue, we initially evaluated the hypothalamic tissue immediately and skeletal muscle and blood samples 3 hours after an acute exercise session, as illustrated (Figure 4A). High levels of $1 / 6$ gene expression were found immediately after the acute aerobic exercise (Figure 4B). In addition, ERK1/2 phosphorylation was increased in whole hypothalamic tissue (Figure S4B), particularly in VMH (Figure 4C). Samples obtained 3 hours after the physical exercise revealed high levels of serum IL6 (Figure 4D). In parallel, AMPK and ACC phosphorylation were still increased in the soleus muscle (Figure S4C). Similar findings were observed in AMPK and ACC phosphorylation levels post-exercise period in the skeletal muscle of rodents ${ }^{25-27}$ and humans ${ }^{28-30}$. RNA-sequencing analysis was performed to confirm the presence of the oxidative profile in the skeletal muscle 3 hours after the acute exercise. Several genes involved in the mitochondrial function, lipid metabolism, and adrenergic signaling were upregulated 3 hours after the acute exercise (Figure 4E), whereas, among 99 genes that were upregulated in response to exercise, almost half of these genes are related to the mitochondrial activity (24 genes), lipid metabolism (15 genes) and adrenergic signaling ( 7 genes) (Figure 4E and F). Remarkably, in contrast to wild-type mice, the same exercise protocol failed to stimulate ERK1/2 phosphorylation in the hypothalamus and flopped to sustain ACC and AMPK phosphorylation 3h post-exercise in the muscle of IL6-null mice (IL6KO) (Figure S4D-F).

To explore the relevance of IL6 action specifically in the hypothalamus, exercised mice received anti-IL6 antibody (IL6 AB) icv microinjections 30 min before and immediately after the exercise session, and the muscle samples were analyzed 3 hours later, as illustrated in the experimental design (Figure S5A). This experiment revealed that anti-IL6 AB icv microinjections abolished the fatty acid oxidation post-exercise in the skeletal muscle (Figure S5B). We confirmed these preliminary findings by employing a versatile tool for genome editing, targeting IL6 receptor (IL6R) specifically in VMH of mice using lentiviral vector (shRNA) microinjection. Five distinct lentiviruses for inhibiting hypothalamic IL6R were tested. The TRCN94 lentivirus was the most efficient, reducing hypothalamic IL6R protein content by approximately $40 \%$ compared to scramble vector (Figure S6A). Next, bilateral microinjections of TRCN94 were then 
performed to deliver IL6R lentivirus specifically in VMH (IL6R KD VMH $)$. Three days after the lentivirus transduction, food consumption was normalized, and no difference was found in terms of food intake between scramble and IL6R KDVMH mice (Figure S6B). Importantly, we confirmed that this lentiviral transduction was restricted to $\mathrm{VMH}$, reducing the IL6R content in $\mathrm{VMH}$, without affecting the presence of this receptor in adjacent hypothalamic nuclei, including in the arcuate nucleus (Figure 4G-J). Seven days after lentivirus microinjection in $\mathrm{VMH}$, the animals were submitted to an acute exercise session. The double-staining assay demonstrated that exercise increased the phospho-ERK1/2 positive cells in VMH in the scramble group (Figure 4K and L). However, exercise did not promote the same effect in IL6R KDVMH mice (Figure 4K and L). Interestingly, high levels of AMPK and ACC phosphorylation were observed 3 hours after exercise in the soleus muscle of control (scramble) but not in IL6R KDVMH mice (Figure $4 \mathrm{M})$. Finally, mass spectrometry analysis performed 3 hours after the exercise protocol confirmed that exercise markedly reduced the amount of fraction of saturated, monosaturated, and polyunsaturated fatty acids fractions in the skeletal muscle of control (scramble), but not in IL6R KD ${ }^{\mathrm{VMH}}$ mice (Figure $4 \mathrm{~N}$ and 6SC).

Altogether, our findings demonstrate that the hypothalamic IL6/ERK axis in VMH drives the fatty acid metabolism in the skeletal muscle (Figure S6D), and this neuromuscular circuitry seems to be critical to underpin the fatty acid oxidation post-exercise.

\section{Declarations}

\section{Acknowledgments}

The authors thank Dr. Johan Auwerx from EPFL, Switzterland, for the assistance for the bioinformatics analysis. Ney Robson de Almeida from the University of São Paulo (USP) for maintaining the a2AC ARKO mice. Dr. Guilherme Z. Rocha, Dr. Luciene Lenhare, Dr. Kellen Cristina da Cruz Rodrigues and Juliana A. Camargo for technical assistance. This work was supported by grants from the São Paulo Research Foundation- FAPESP scholarships (2012/23006-1 and 2013/26053-3), research project (2019/21709-4), Brazilian National Council for Scientific and Technological Development - CNPq (477478/2012-3) and Coordination for the Improvement of Higher Education Personnel (CAPES), number 001. L.J.E.G. was supported by the European Union's Horizon 2020 research and innovation programme through the Marie Skłodowska-Curie Individual Fellowship "AmyloAge" (grant agreement No. 896042).

\section{Author contributions}

C.K.K. and T.O.M. performed the Western blot analysis. C.K.K., T.O.M., and V.R.R.S. performed oxygen consumption and PCR analysis. A.M.A. performed the immunofluorescence and lentivirus assays. M.R.T. and F.M.S. performed the N2A cells experiments. C.K.K. performed the denervation experiments. C.K.K and B.M.C. performed stereotaxy surgeries and blue dye sataining. C.O.R. and D.E.C performed mass spectrometry analysis. R.S.G. and E.R.R. performed the bioinformatics analysis. T.O.M., P.P., D.G.T., M.C.G. A.L.R., and S.T. contributed to all IL6 knockout experiments. T.O.M., S.M.H., and J.C.R.N. performed fatty 
acid oxidation assays. R.S.G. performed the RNAseq analysis. L.J.EG. performed the bioinformatics analysis in figure S2C and B. P.C.B. provided a2AC AR knockout mice. P.O.P. and R.R.B. conducted the VMH dissection experiment. R.C., A.S.R.S., L.P.M., J.R.P., D.E.C, and L.A.V. provided the laboratory support and contributed to the discussion. E.R.R. wrote the manuscript.

\section{Declaration of interests}

All authors declared no competing interests.

\section{References}

1. Wallenius, V. et al. Interleukin-6-deficient mice develop mature-onset obesity. Nat. Med. 8, 75-9 (2002).

2. Timper, K. et al. IL-6 Improves Energy and Glucose Homeostasis in Obesity via Enhanced Central IL6 trans-Signaling. Cell Rep. 19, 267-280 (2017).

3. Kim, K. W. et al. FOXO1 in the ventromedial hypothalamus regulates energy balance. J. Clin. Invest. 122, 2578-89 (2012).

4. Shiuchi, T. et al. Hypothalamic orexin stimulates feeding-associated glucose utilization in skeletal muscle via sympathetic nervous system. Cell Metab. 10, 466-80 (2009).

5. Gavini, C. K., Jones, W. C. \& Novak, C. M. Ventromedial hypothalamic melanocortin receptor activation: regulation of activity energy expenditure and skeletal muscle thermogenesis. J. Physiol. 594, 5285-5301 (2016).

6. Minokoshi, Y. et al. Leptin stimulates fatty-acid oxidation by activating AMP-activated protein kinase. Nature 415, 339-43 (2002).

7. Lonsdale, J. et al. The Genotype-Tissue Expression (GTEx) project. Nat. Genet. 45, 580-585 (2013).

8. Andreux, P. A. et al. Systems Genetics of Metabolism: The Use of the BXD Murine Reference Panel for Multiscalar Integration of Traits. Cell 150, 1287-1299 (2012).

9. Shiuchi, T. et al. Hypothalamic Orexin Stimulates Feeding-Associated Glucose Utilization in Skeletal Muscle via Sympathetic Nervous System. Cell Metab. 10, 466-480 (2009).

10. Fujikawa, T. et al. SF-1 expression in the hypothalamus is required for beneficial metabolic effects of exercise. Elife 5, (2016).

11. Toda, C. et al. Extracellular signal-regulated kinase in the ventromedial hypothalamus mediates leptin-induced glucose uptake in red-type skeletal muscle. Diabetes 62, 2295-307 (2013). 
12. Strickland, J. et al. Interleukin-6 increases the expression of key proteins associated with steroidogenesis in human NCl-H295R adrenocortical cells. Steroids 119, 1-17 (2017).

13. Fang, X. X., Jiang, X. L., Han, X. H., Peng, Y. P. \& Qiu, Y. H. Neuroprotection of interleukin- 6 against NMDA-induced neurotoxicity is mediated by JAK/STAT3, MAPK/ERK, and PI3K/AKT signaling pathways. Cell. Mol. Neurobiol. 33, 241-251 (2013).

14. Rahmouni, K., Sigmund, C. D., Haynes, W. G. \& Mark, A. L. Hypothalamic ERK mediates the anorectic and thermogenic sympathetic effects of leptin. Diabetes 58, 536-542 (2009).

15. Tanida, M. et al. Hypothalamic nesfatin-1 stimulates sympathetic nerve activity via hypothalamic ERK signaling. Diabetes 64, 3725-3736 (2015).

16. Jankord, R. et al. Stress activation of IL-6 neurons in the hypothalamus. Am. J. Physiol. - Regul. Integr. Comp. Physiol. 299, (2010).

17. Bongartz, H., Seiß, E. A., Bock, J. \& Schaper, F. Glucocorticoids attenuate interleukin-6-induced c-Fos and Egr1 expression and impair neuritogenesis in PC12 cells. J. Neurochem. 157, 532-549 (2021).

18. Cha, S. H., Hu, Z., Chohnan, S. \& Lane, M. D. Inhibition of hypothalamic fatty acid synthase triggers rapid activation of fatty acid oxidation in skeletal muscle. Proc. Natl. Acad. Sci. U. S. A. 102, 1455714562 (2005).

19. Kimber, N. E., Cameron-Smith, D., McGee, S. L. \& Hargreaves, M. Skeletal muscle fat metabolism after exercise in humans: Influence of fat availability. J. Appl. Physiol. 114, 1577-1585 (2013).

20. Henderson, G. C. \& Alderman, B. L. Determinants of resting lipid oxidation in response to a prior bout of endurance exercise. J. Appl. Physiol. 116, 95-103 (2014).

21. Malatesta, D., Werlen, C., Bulfaro, S., Chenevière, X. \& Borrani, F. Effect of high-intensity interval exercise on lipid oxidation during postexercise recovery. Med. Sci. Sports Exerc. 41, 364-374 (2009).

22. Nadermann, N. \& Volkoff, H. Effects of short-term exercise on food intake and the expression of appetite-regulating factors in goldfish. Peptides 123, 170182 (2020).

23. Ropelle, E. R. et al. IL-6 and IL-10 anti-inflammatory activity links exercise to hypothalamic insulin and leptin sensitivity through IKK $\beta$ and ER stress inhibition. PLoS Biol. 8, (2010).

24. Nybo, L., Nielsen, B., Pedersen, B. K., Møller, K. \& Secher, N. H. Interleukin-6 release from the human brain during prolonged exercise. J. Physiol. 542, 991-5 (2002).

25. Liu, X., Niu, Y., Yuan, H., Huang, J. \& Fu, L. AMPK binds to Sestrins and mediates the effect of exercise to increase insulin-sensitivity through autophagy. Metabolism. 64, 658-665 (2015). 
26. He, C. et al. Exercise-induced BCL2-regulated autophagy is required for muscle glucose homeostasis. Nature 481, 511-515 (2012).

27. Pauli, J. R. et al. Acute physical exercise reverses S-nitrosation of the insulin receptor, insulin receptor substrate 1 and protein kinase B/Akt in diet-induced obese Wistar rats. J. Physiol. 586, (2008).

28. Kjøbsted, R. et al. Intact regulation of the AMPK signaling network in response to exercise and insulin in skeletal muscle of male patients with type 2 diabetes: Illumination of AMPK activation in recovery from exercise. Diabetes $65,1219-1230$ (2016).

29. Norrbom, J. et al. Alternative splice variant PGC-1 a-b is strongly induced by exercise in human skeletal muscle. Am. J. Physiol. - Endocrinol. Metab. 301, (2011).

30. Lee-Young, R. S. et al. Carbohydrate ingestion does not alter skeletal muscle AMPK signaling during exercise in humans. Am. J. Physiol. - Endocrinol. Metab. 291, (2006).

\section{Figures}



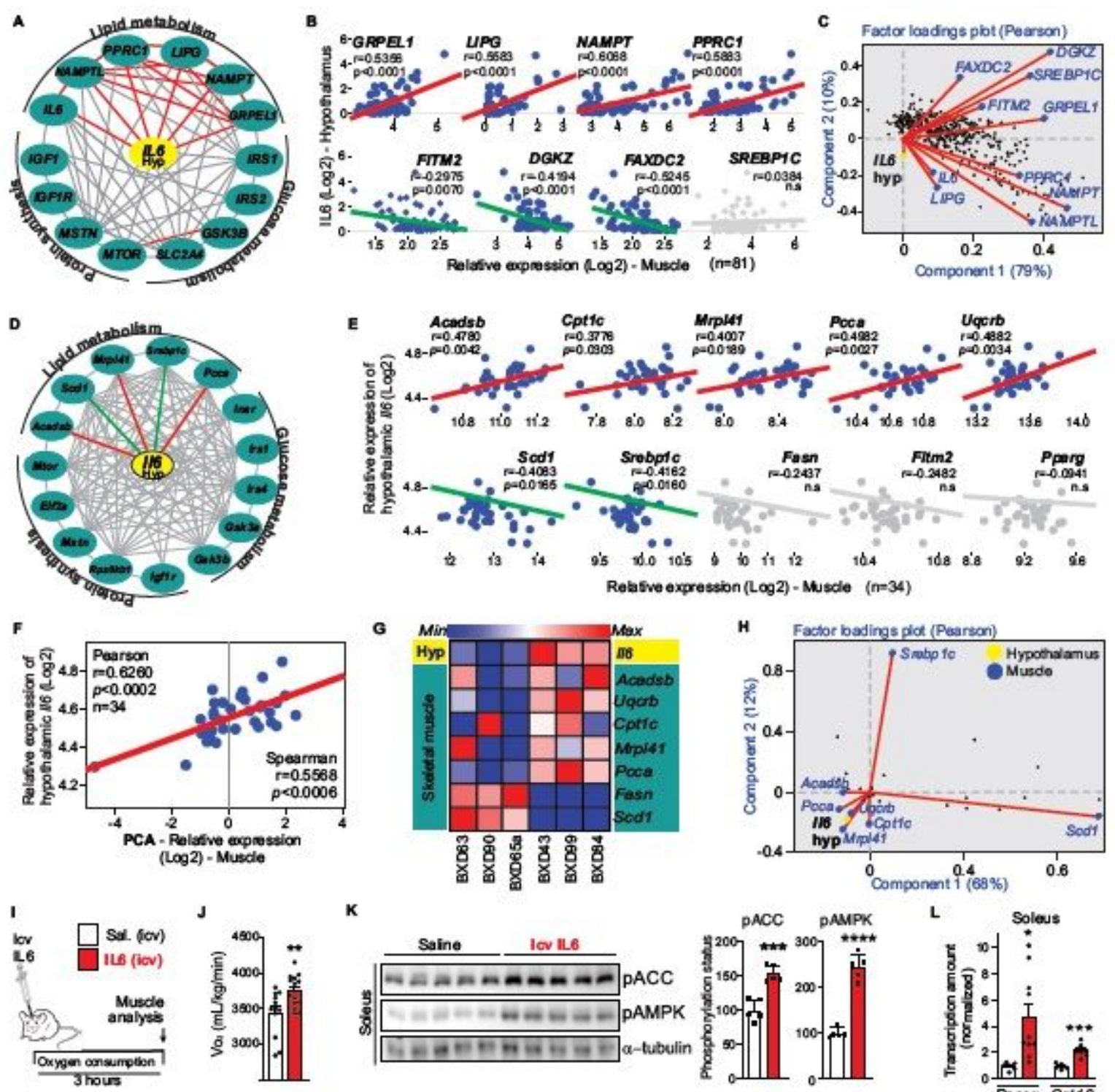

K
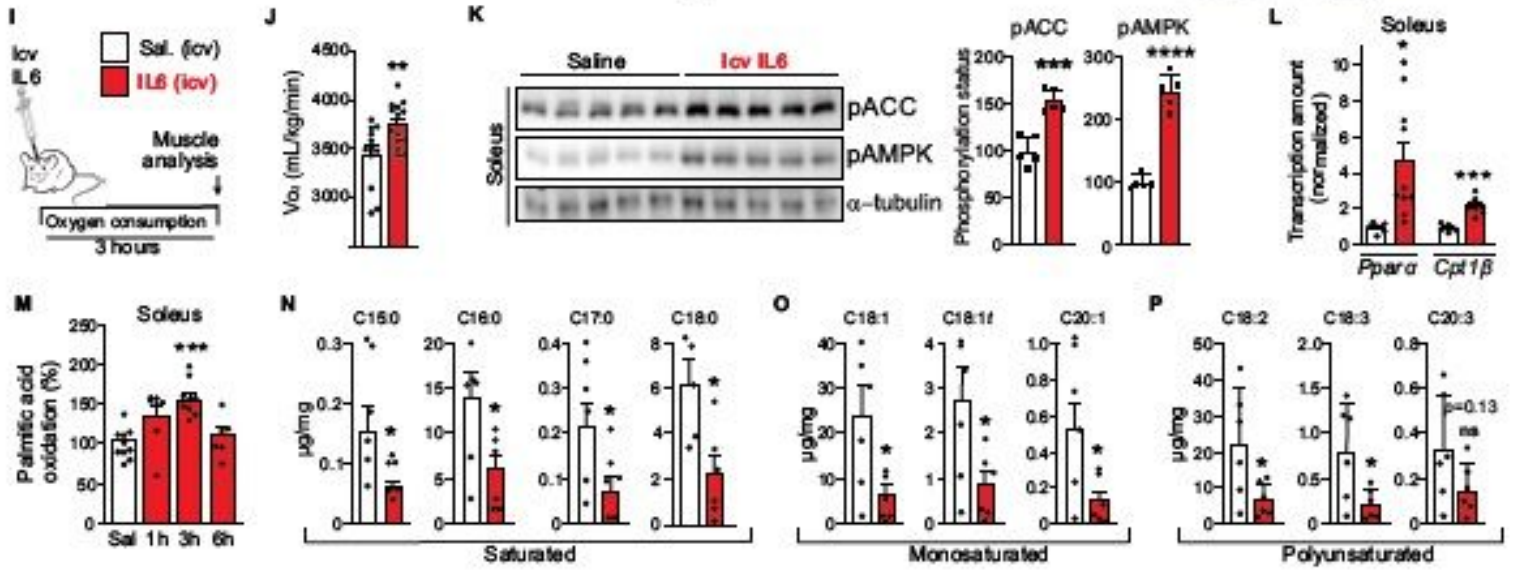

\section{Figure 1}

Hypothalamic interleukin-6 induces fatty acid oxidation in muscle (A) Interactome and (B) Pearson correlation graphs show the correlation between hypothalamic IL6 gene expression and several genes of human skeletal muscle. Red lines represent positive and green lines represent negative correlations. Grey line represents no correlation. Pearson's correlation $r \geq|0.5|$ and $p<0.05$. $(n=81)$. (C) Factor loading plot (biplot) angles more than $90^{\circ}$ between gene vectors represent a negative correlation. (D) Interactome and (E) Pearson's correlation graphs show the correlation between hypothalamic Il6 gene expression and several genes of human skeletal muscle of genetically diverse BXD mice. Red lines represent positive and 
green lines represent negative correlations. Grey lines represent no correlation. Pearson's correlation $r \geq$ |0.35| and p<0.05. ( $n=34)$. (F) PCA value was obtained using Acadsb, Cpt1c, Mrpl41, Pcca, and Uqcrc relative expression in muscle of BXD mice. Pearson's and Spearman's analysis shows the positive correlation between PCA and hypothalamic 116 gene expression in BXD mice. (G) Heat map graph using hypothalamic and muscle transcripts from 6 different BXD mice strains. $(\mathrm{H})$ Factor loading plot (biplot) by using BXD cohort. (I) Experimental design using male C57BL6/j mice. $(\mathrm{J})$ Oxygen consumption during 3 hours after icv IL6 recombinant microinjection (200ng) ( $n=11-14, * \star p<0.01$ vs saline group). (K) ACC Ser79/Ser212 and AMPK Thr172 phosphorylation in the soleus muscle of C57BL6/j mice ( $n=5)$. (L) Cpt1 and Ppar $\delta$ mRNA levels were determined using real-time PCR assay in the soleus muscle $3 \mathrm{~h}$ after recombinant IL6 icv microinjection ( $n=6-10)$. (M) Palmitic acid oxidation assay in the soleus muscle in mice injected with IL6 ( $n=6-10)$. (N-P) Determination of fatty acid fractions using mass spectrometry analysis in the gastrocnemius muscle $3 \mathrm{~h}$ after recombinant IL6 icv microinjection. Unpaired t-test was used in $\mathrm{J}, \mathrm{K}, \mathrm{L}, \mathrm{N}, \mathrm{O}$ and $\mathrm{P}$. One-way analysis of variance was used for statistical analysis in $\mathrm{M} .\left({ }^{*}, \mathrm{p}<0.05\right.$, $\star *, p<0.01, * \star *, p<0.001$ and ${ }^{* \star * *}, p<0.0001$, vs saline). 

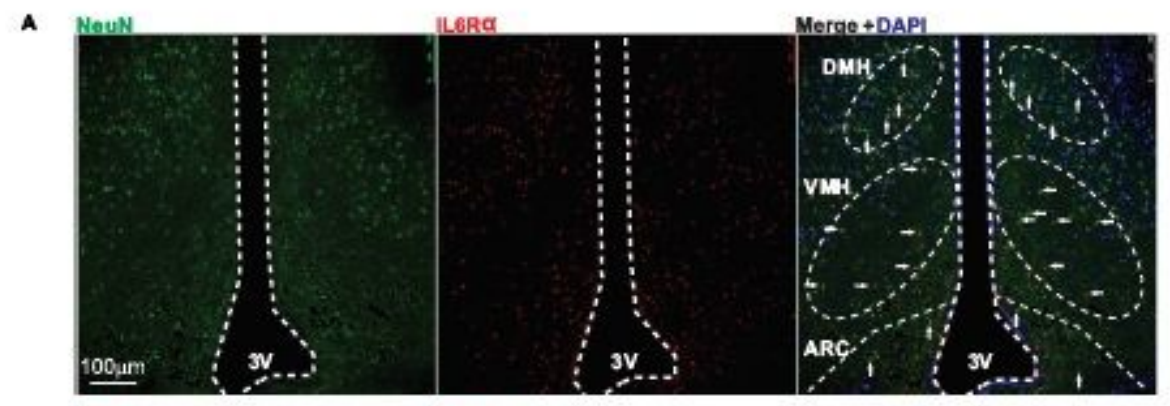

B

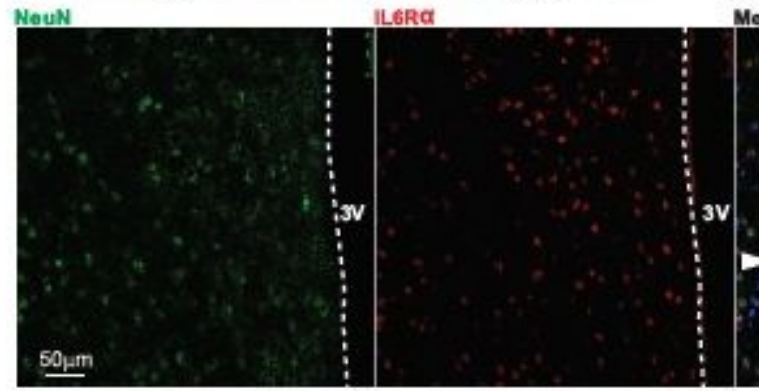

Mergo + DAPI
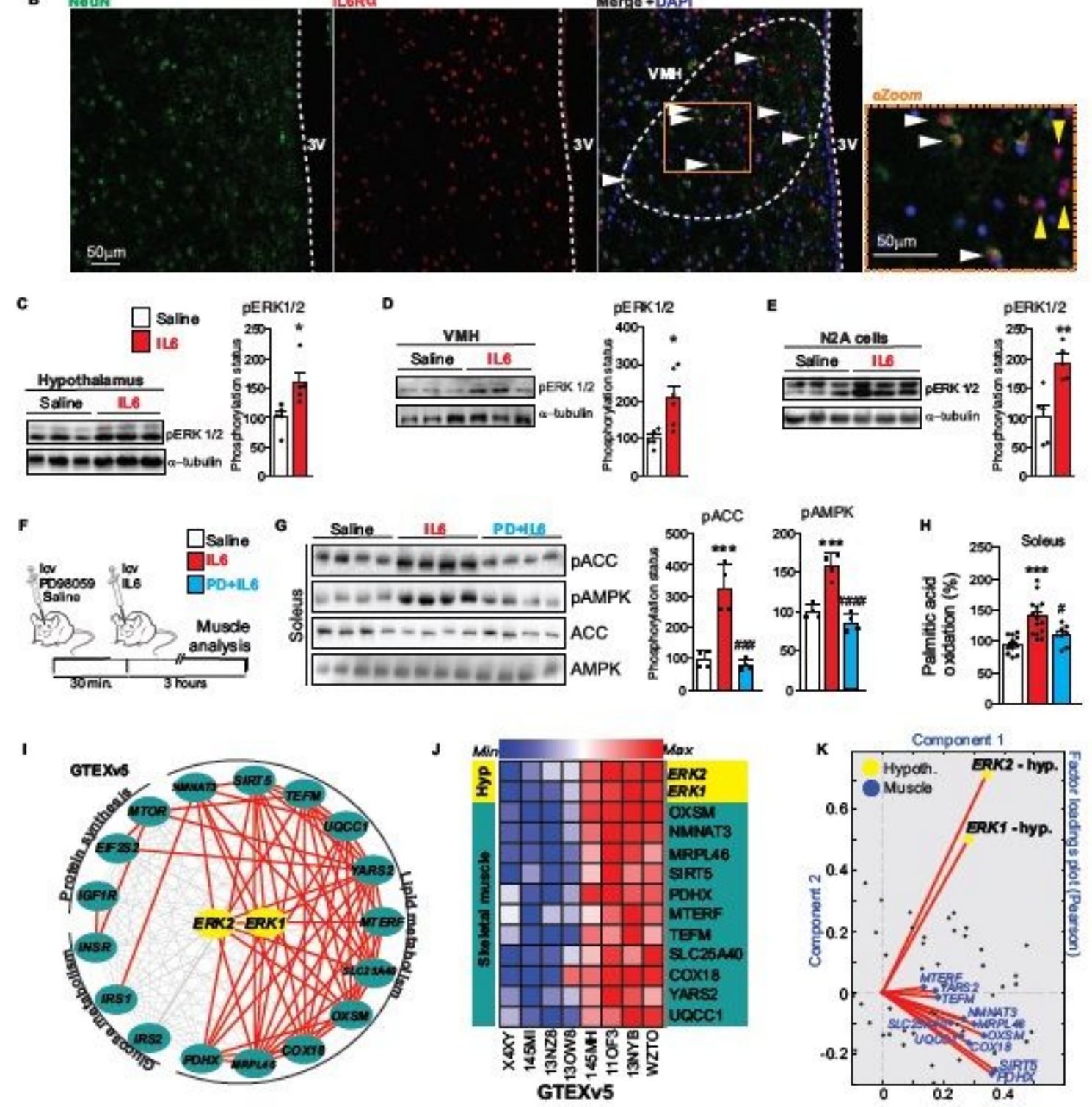

Figure 2

Evaluation of hypothalamic IL6/ERK axis. Immunostaining from the brain of intact male C57BL6/j mice was performed to evaluate colocalization of IL6 receptor (red) in neurons (green) in; (A) hypothalamic nuclei surrounding of the third ventricle (scale bar $=100 \mu \mathrm{m})$ and $(B)$ in ventromedial nuclei $(\mathrm{VMH})$ of mice $(n=5$, scale bar $=50 \mu m)$. Digital/electronic zoom (eZoom) in the right side of figure $B$ was performed (scale bar $=50 \mu \mathrm{m}$ ). Western blotting assays show; (C) ERK 1/2 Thr202/Tyr204 
phosphorylation 30 minutes after recombinant IL6 icv microinjection (200ng) in the whole hypothalamus $(n=5)$ and in (D) in the ventromedial nucleus $(n=5-6)$. (E) ERK1/2 Thr202/Tyr204 phosphorylation in Neuro2A cells after recombinant IL6 incubation $(50 \mathrm{ng} / \mathrm{mL})$ for $60 \mathrm{~min}(\mathrm{n}=5)$. (F) Schematic view of the experiment in which a group of mice received icv microinjection of PD $98059(60 \mu \mathrm{M}), 30$ minutes later they received IL6 (200ng) icv microinjection, and 3 hours afterward, soleus muscles were obtained. (G) Western blot shows ACC Ser79/Ser212 and AMPK Thr172 phosphorylation in soleus muscle $(n=4)$. $(H)$ Palmitic acid oxidation in the soleus muscle $(n=8-15)$. (I) Interactome and (G) Pearson's correlation graphs show the positive correlation between hypothalamic ERK1 and ERK2 gene expression and genes related to lipid, glucose, and protein metabolism in the skeletal muscle of humans. Red lines represent positive, and grey lines represent no correlation. Pearson's $r \geq|0.35|$ and $p<0.05$. $(n=81)$. (H) Factor loadings plot shows that hypothalamic ERK1 and ERK2 gene expressions positively correlate with genes related to fatty acid oxidation in the human muscle as seen on a factor loading plot (biplot), where angles less than $90^{\circ}$ between gene vectors represent a positive correlation. Unpaired t-test was used in $C$, $D$, and E. One-way analysis of variance was used for statistical analysis in $G$ and $H .(*, p<0.05, * *, p<0.01$, and

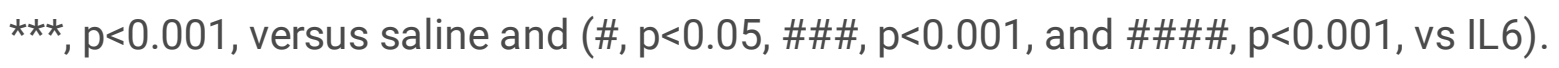



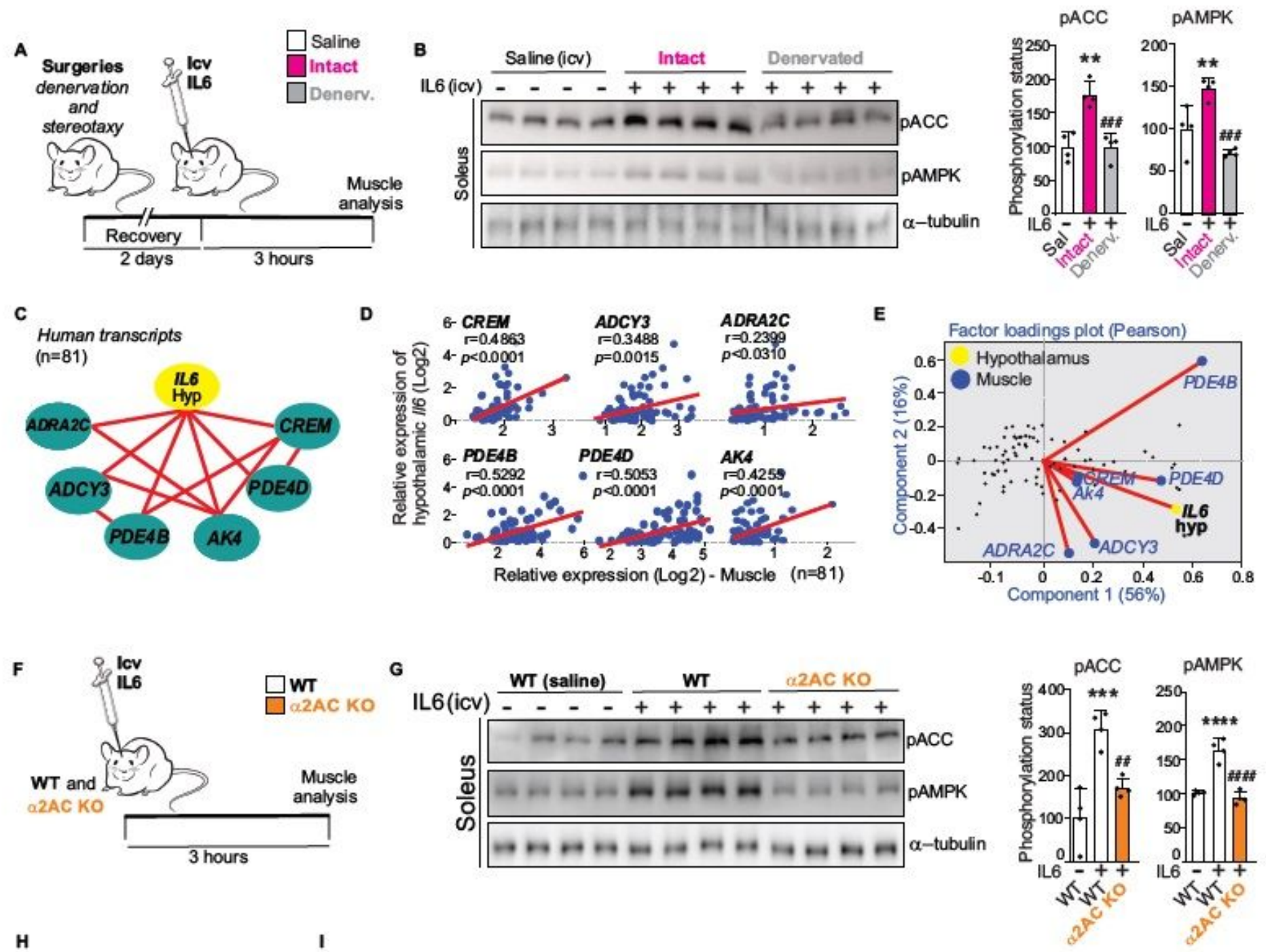

H

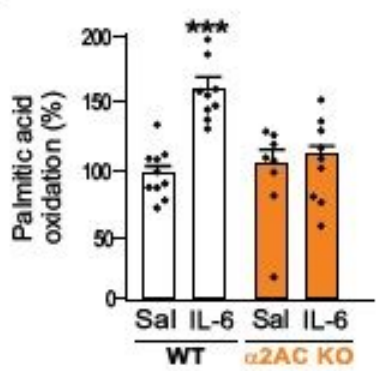

I

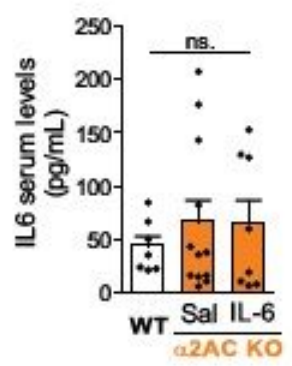

\section{Figure 3}

Evaluation of a2A/a2C-adrenoceptor on fatty acid oxidation in muscle. (A) Experimental design. The denervation (sciatic nerve) and the cannulae implantation in the third ventricle were performed on the same procedure day. (B) Western blot shows ACC and AMPK phosphorylation in soleus muscle $3 \mathrm{~h}$ after icv infusion of IL6 (200ng) ( $n=4, * \star, p<0.01$ vs saline and \#\#\#, $p<0.001$ vs intact+IL6). (C) The interaction graph shows the positive correlation (red lines) between hypothalamic IL6 gene expression and the gene set related to alpha-adrenergic signaling in the muscle of humans. (D) Pearson's correlation was performed using GTEXv5 human brain hypothalamus Refseq (Sep 15) RPKM Log2 and GTEXv5 human muscle-skeletal Refseq (Sep 15) RPKM Log2 datasets. Red lines represent positive correlation ( $n=81$, 
$p<0.031)$. (E) Factor loading plot (biplot) using the same datasets. (F) Male a2A/a2C-adrenoceptor knockout mice were injected with icv IL6 recombinant, and 3 hours later, the skeletal muscle was examined. (G) Western blot shows ACC Ser79/Ser212 and AMPK Thr172 phosphorylation in soleus muscle $3 \mathrm{~h}$ after icv infusion of IL6 (200ng) ( $n=4, * \star \star, p<0.001$ and $* \star \star \star, p<0.0001$ vs wild-type plus saline and \#\#, $p<0.01$ and \#\#\#\#, $p<0.0001$ vs wild-type plus IL6). (H) Palmitic acid oxidation ( $n=8-10$, ***, $p<0.001$ vs wild-type plus saline). (I) Serum levels of IL6 ( $n=7-11$, ns, non-significant). One-way analysis of variance was used for statistical analysis in $B, G, H$, and I.
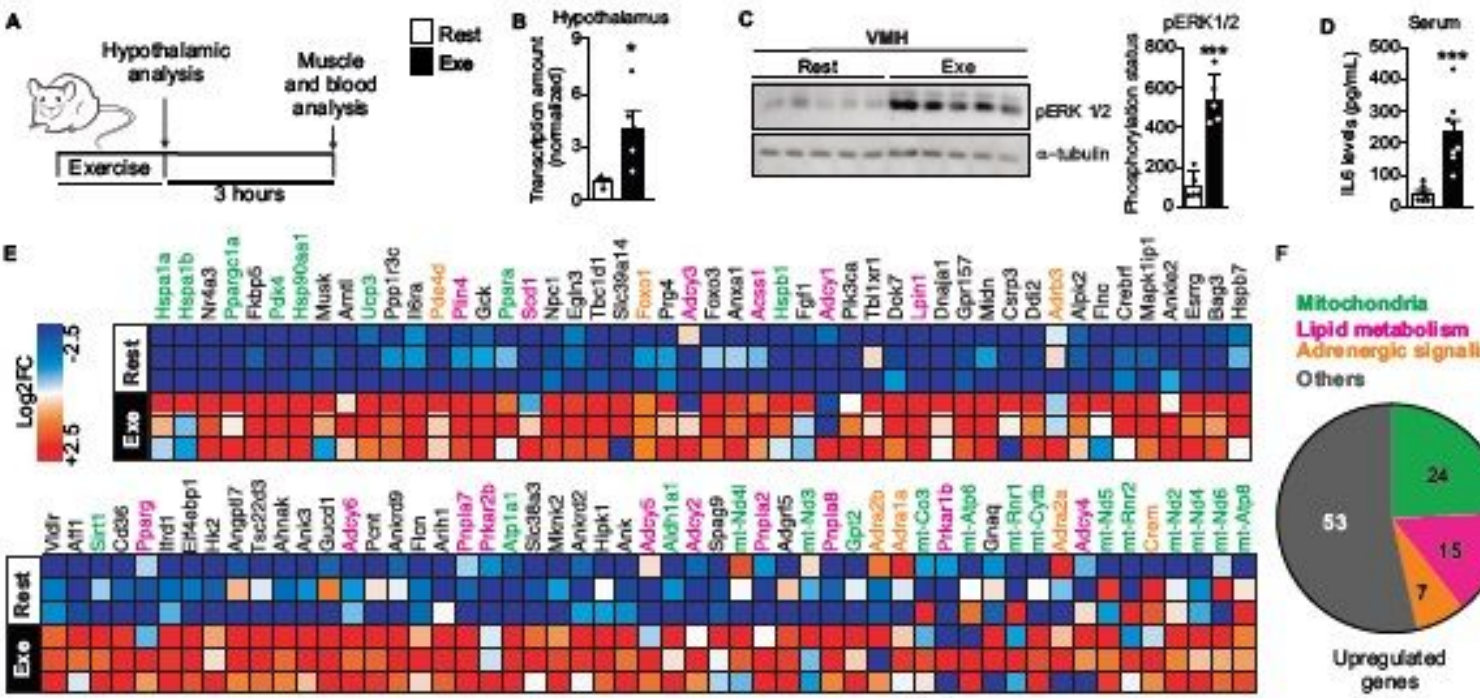

F
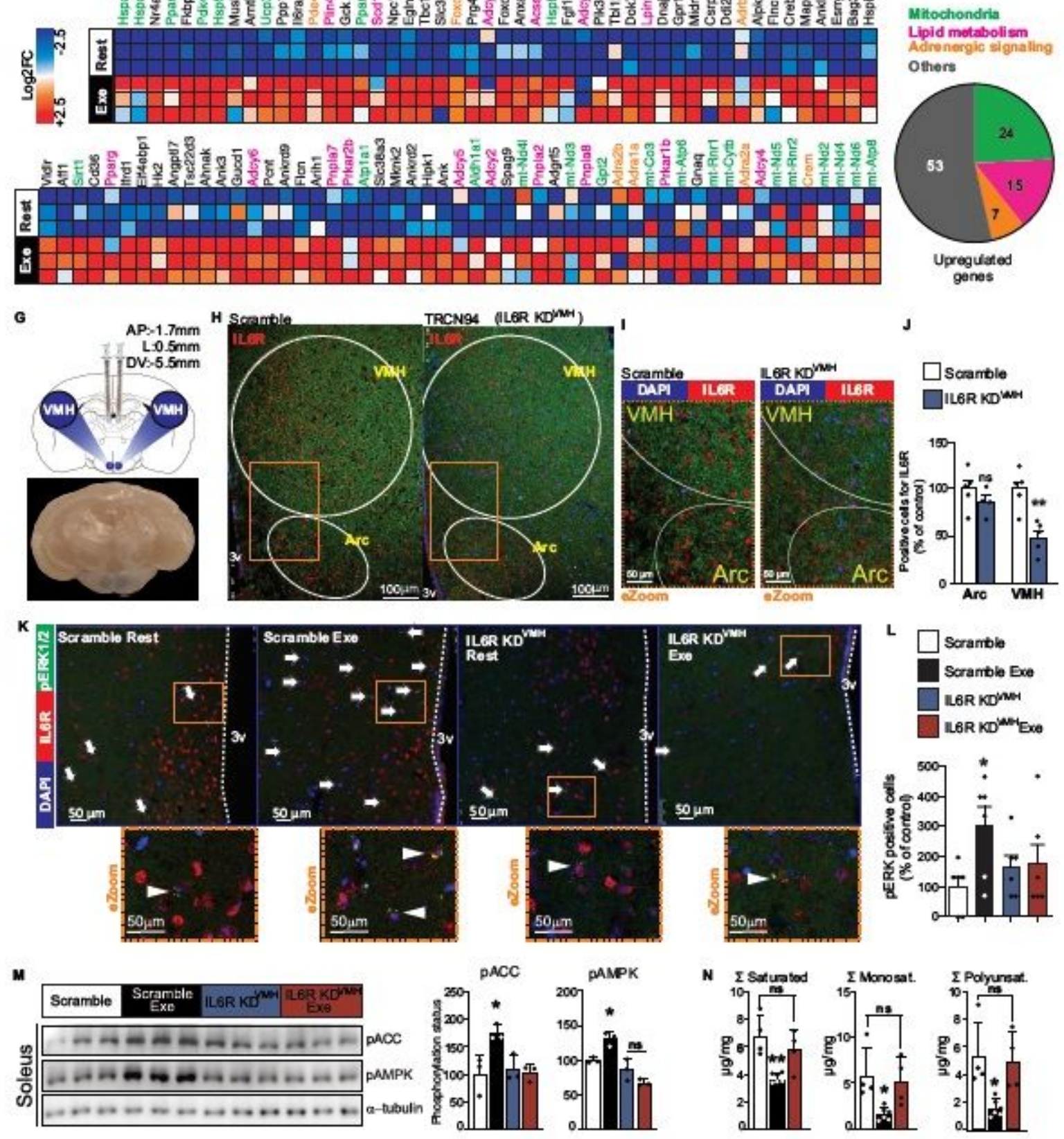


\section{Figure 4}

Hypothalamic IL6 controls muscle fatty acid oxidation post-exercise in mice. (A) Experimental design. (B) Hypothalamic 116 gene expression in control and exercised mice $\left(n=4-6,{ }^{*} p<0.05\right.$ versus control group at rest). (C) ERK1/2 phosphorylation in VMH, immediately after acute exercise $(n=5)$. (D) Serum levels of IL6 in control and exercised mice ( $n=7-8, \star \star \star p<0.001$ vs control group). (E) RNAseq assay was performed to evaluate the skeletal muscle signature in gene expression in the skeletal muscle 3 hours after the acute exercise in mice $(n=3)$. (F) Distribution and quantification of upregulated genes in the skeletal muscle by function in the RNAseq assay. (G) Illustration for VMH microinjections containing Scramble or IL6 Receptor KD lentivirus, AP-antero-posterior, L-Lateral, and DV-Dorsoventral (upper panel) and representative picture of a coronal view of a mouse brain, demonstrating the blue dye staining confirming the anatomical localization of bilateral microinjections in VMH (lower panel). (H) Immunostaining of IL6 receptor (red) in the arcuate nucleus (Arc) and ventromedial hypothalamus (VMH) 7 days after Scramble or IL6 Receptor KD lentivirus transfection in VMH of mice $(n=5-6$, scale bar $100 \mu \mathrm{m})$. (I) Electronic zoom (eZoom) from the orange rectangles highlighted in figure $\mathrm{H}$ were performed, showing the presence of IL6R (red) in the arcuate nucleus (Arc) and ventromedial hypothalamus (VMH) (scale bar $=50 \mu \mathrm{m}) .(\mathrm{J})$ IL6R positive cells in the arcuate and ventromedial nuclei of mice $(n=5-6, * * p<0.01$ vs scramble). (K) Immunostaining 7 days after Scramble or IL6 Receptor KD lentivirus transfection in VMH of mice at rest or after acute exercise. Arrows indicate the presence of ERK1/2 phosphorylation in $\mathrm{VMH}(n=5$, Scale bar $50 \mu \mathrm{m}$ ), upper panels. (K, in lower panels) Digital/electronic zoom (eZoom) from the orange rectangles highlighted in figure $\mathrm{K}$ (upper panels) were performed, showing the presence of ERK1/2 phosphorylation in VMH (scale bar $=50 \mu \mathrm{m}$ ). (L) Quantification of positive cells for ERK1/2 phosphorylation in VMH of mice ( $n=6,{ }^{*}<<0.05$ vs scramble at rest). (M) Western blot shows ACC Ser79/Ser212 and AMPK Thr172 phosphorylation in soleus muscle $3 \mathrm{~h}$ after the exercise protocol $(n=3, *, p<0.05$ vs scramble at rest). (J) Determination of fatty acid fractions using mass spectrometry analysis in the gastrocnemius muscle $3 \mathrm{~h}$ after the acute exercise $(n=4-6)$. Unpaired t-test was used in B, C, D, J, and L. One-way analysis of variance was used for statistical analysis in $\mathrm{M}$ and $\mathrm{N}$.

\section{Supplementary Files}

This is a list of supplementary files associated with this preprint. Click to download.

- KatashimaSupplementayInformationFINAL.docx

- KatashimaetalFigureS1.pdf

- KatashimaetalFigureS2.pdf

- KatashimaetalFigureS3.pdf

- KatashimaetalFigureS4.pdf

- KatashimaetalFigureS5.pdf

- KatashimaetalFigureS6.pdf 